\title{
Total scattering at grazing incidence to study real thin film systems at variable temperature
}

\author{
A.-C. Dippel' ${ }^{1}$, O. Gutowski ${ }^{1}$, M. Roelsgaard'², B. B. Iversen², M. Sturm ${ }^{1}$, M. v. Zimmermann ${ }^{1}$ \\ ${ }^{1}$ Deutsches Elektronen-Synchrotron DESY, Notkestrasse 85, 22607 Hamburg, Germany, ${ }^{2}$ Department of Chemistry, Aarhus \\ University, Langelandsgade 140, 8000 Aarhus C, Denmark
}

ann-christin.dippel@desy.de

Complementary to x-ray diffraction patterns that represent the crystal lattice in $Q$ space, the atomic pair distribution function (PDF) describes the structure of a material as a histogram of interatomic distances $r$ in real space. The total scattering (TS) approach that enables PDF analysis requires that scattering data is collected over a wide $Q$ range of the order of $20 \AA^{-1}$ and subsequent Fourier transformation of the entire scattering pattern into direct space. While TS at high-energy beamlines has become a standard routine for bulk-type samples, the unfavorable thickness ratio of a thin film (nanometer regime) to its substrate (micrometer regime) limits the detectability of the film signal in simple transmission geometry as described e.g. in Ref. [1]. Therefore, we applied the high-energy surface diffraction technique established for single-crystal surfaces [2] to less ordered films and thus pushed the capabilities for PDF analysis of thin films to unprecedented limits in terms of minimum thickness and time resolution. [3,4] Besides polycrystalline and textured metal and oxide layers, we studied amorphous and naocrystalline thin films. By careful data treatment, we successfully derived PDFs of comparable data quality from different $\mathrm{HfO}_{2}$ films with thicknesses down to $15 \mathrm{~nm}$ independent on their degree of ordering with domain sizes between $\sim 5$ and $>30 \AA$. All films were deposited on fused silica which provides an easily scalable background to subtract from the sample data to isolate the film signal. Real thin film devices e.g. for electronic applications, however, typically consist of multiple layers, and the film growth is largely affected by the nature of the underlying layer. Therefore, we further developed grazing incidence total scattering towards a depth-resolving method by scanning the incidence angle. In this way, the technique provided insight into the structure of different types of bilayer samples studied for their use e.g. in next-generation computer memory applications. PDFs were successfully extracted from the individual layers of different combinations and stackings of amorphous and crystalline materials exhibiting high and low (electron) density and, hence, $\mathrm{x}$-ray scattering power from $\mathrm{TiO}_{2}$ to $\mathrm{Pt}[5]$. As thermal treatment is an essential part of thin film device manufacturing, we are developing a laser-interferometer based system that, beyond data collection during isothermal heat-treatment as applied in [4], enables following structural changes during variabletemperature processes up to several hundred degrees. Fig. 1 shows data from the proof-of-concept experiment on a $30 \mathrm{~nm} \mathrm{HfO}_{2}$ thin film deposited by chemical vapor deposition in an amorphous state, crystallized in situ while continuously acquiring TS data.

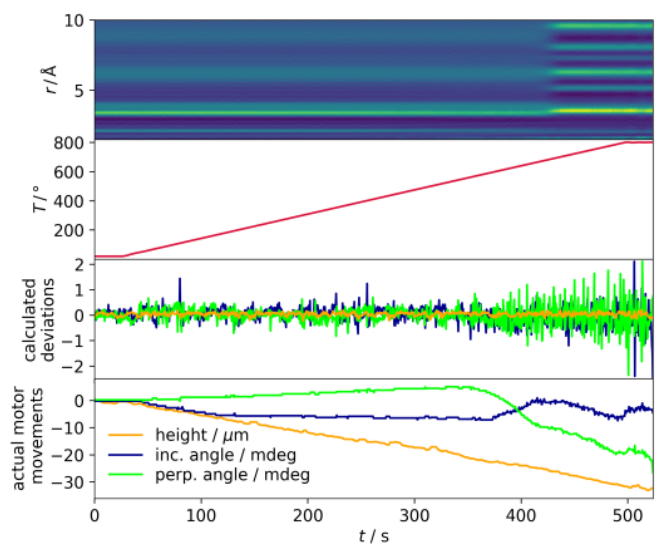

Figure 1. PDFs of an amorphous hafnium oxide film acquired during crystallization at the given heating rate of $100 \mathrm{~K} \mathrm{~min}^{-1}$, incl. the fluctuations of the sample and the actual motor movements necessary to compensate for the thermal expansion.

[1] Jensen, K. M. Ø., Blichfeld, A. B., \& Goldman, A. I. (1999). Rev. Sci. Instrum. 70, 3554.

[2] Gustafson, J., Shipilin, M., Zhang, C., Stierle, A., Hejral, U., Ruett, U., Gutowski, O., Carlsson, P.-A., Skoglundh, M., Lundgren, E. (2014). Science 343, 758.

[3] Dippel, A.-C., Roelsgaard, M., Böttger, U., Schneller, T., Gutowski, O., Rütt, U. (2019). IUCrJ 6, 290.

[4] Roelsgaard, M., Dippel, A.-C., Borup, K. A., Nielsen, I. G., Broge, N. L. N., Röh, J. T., Gutowski, O., Iversen, B. B. (2019). IUCrJ 6, 299.

[5] Dippel, A.-C., Gutowski, O., Klemeyer, L., Boettger, U., Berg, F., Schneller, T., Hardtdegen, A., Aussen, S., Hoffmann-Eifert, S., Zimmermann, M. v. (2020). Nanoscale 12, 13103.

Keywords: total scattering; pair distribution function; thin films; grazing incidence

Acta Cryst. (2021), A77, C266 